\title{
Population-Based Analysis of Sudden Death in Children: The Oregon Sudden Unexpected Death Study
}

\author{
Sumeet S. Chugh, Kyndaron Reinier, Seshadri Balaji, Audrey Uy-Evanado, Cathy Vickers, \\ Ronald Mariani, Karen Gunson, and Jonathan Jui \\ Heart Institute, Cedars-Sinai Medical Center, Los Angeles CA; and the Departments of Emergency \\ Medicine, Pediatrics and Pathology, Oregon Health and Science University, Portland OR
}

\begin{abstract}
Background-There is a lack of prospective population-based data regarding sudden death in children.

Objective - To assess the burden of sudden cardiac arrest (SCA) in the pediatric population in a 3year community-wide study.

Methods-During 2002-2005, all residents of Multnomah County, Oregon (Pop. 660,486) who suffered SCA were ascertained from emergency medical services, the medical examiner and emergency rooms of 16 area hospitals. A comprehensive evaluation was performed, including analysis of circumstances of death, medical records and available autopsy data. Annual incidence rates were calculated for all residents age $<18$ years, using the 2000 US Census data.

Results-A total of 33 children met criteria for SCA (58\% female, median age $0.37 \mathrm{yr}$, range $0.03-12.3 \mathrm{yr})$. The majority of SCAs (76\%) occurred in children age $<1 \mathrm{yr}$. At least $90 \%$ of this subgroup also met criteria for the sudden infant death syndrome (SIDS). Pediatric SCAs constituted $2.8 \%$ of all SCAs. The pediatric annual incidence rate per 100,000 population was 1.7 (95\% C.I. 1.1 -2.3 ), compared to $60 / 100,000$ for all ages. The pediatric annual incidence rate per 100,000 children was 7.5 (95\% C.I. 5.1 - 10.5). The annual incidence rate of SIDS was $0.8 / 1000$ live births. In contrast to an adult survival rate of $8 \%$, none of the children survived to be discharged from the hospital.
\end{abstract}

Conclusions-The burden of pediatric sudden death was low (3\% of all sudden deaths) but $90 \%$ occurred before age one year and the majority were diagnosed as SIDS (70\% of overall sudden deaths in children). Population education to prevent SIDS and enhanced postnatal diagnosis of occult heart disease are likely to have the greatest impact on prevention of pediatric sudden death.

\section{Keywords}

Sudden cardiac death; screening; pediatric; children; long QT syndrome; incidence; population; sudden infant death syndrome; epidemiology

(C) 2009 The Heart Rhythm Society. Published by Elsevier Inc. All rights reserved.

Address correspondence to: Sumeet S. Chugh MD, The Heart Institute, 5702 South Tower, Cedars-Sinai Medical Center, 8700 Beverly Blvd, Los Angeles, CA 90048; Fax 310-423-3522; sumeet.chugh@cshs.org.

The authors do not have any conflicts of interest.

Publisher's Disclaimer: This is a PDF file of an unedited manuscript that has been accepted for publication. As a service to our customers we are providing this early version of the manuscript. The manuscript will undergo copyediting, typesetting, and review of the resulting proof before it is published in its final citable form. Please note that during the production process errors may be discovered which could affect the content, and all legal disclaimers that apply to the journal pertain. 


\section{INTRODUCTION}

Sudden death in children is a relatively uncommon but devastating problem. There are few prospective population-based studies and the annual incidence is poorly defined. Existing retrospective death-certificate based studies suggest that the annual incidence of sudden death in US children ranges between $0.8-6.2$ per 100,000 population (1-3). A recent one-year prospective, population-based analysis of all-ages sudden cardiac arrest (SCA) in a large US community reported that determination of SCA based on death certificates was both inaccurate (positive predictive value 19\%) as well as an overestimate (4). A better estimation of the magnitude of pediatric sudden death with simultaneous evaluation of specific etiologies is likely to be an essential prerequisite for the deployment of future strategies for prevention and resuscitation.

Since the overall magnitude is small, determination of the epidemiology of pediatric sudden death is likely to require a distinct, focused and intensive effort in a large US subpopulation. As part of the ongoing Oregon Sudden Unexpected Death Study (Ore-SUDS), we performed a prospective, 3-year, community-wide, multiple-source evaluation of pediatric sudden death among the 660,486 residents of Multnomah County, Oregon, USA.

\section{METHODS}

All aspects of this investigation were approved by the Institutional Review Board of Oregon Health and Science University as well as the other health systems providing health care to County residents.

\section{Definition of SCA}

Based on World Health Association criteria, SCA was defined as sudden unexpected collapse within 1 hour of symptom-onset (witnessed) or subjects were to have been observed alive and symptom-free within 24 hours of the event (unwitnessed). Of these, subjects with non-cardiac terminal illness or an identifiable non-cardiac etiology of sudden death or sudden cardiac arrest associated with trauma, violent death, overdose, drowning and suicide were excluded.

\section{Population Evaluated}

The Ore-SUDS study identified all residents of Multnomah County, Oregon (population 660,486) that suffered SCA between Feb 1, 2002 and Jan 31, 2005 from the following multiple sources: the emergency medical response system, the County medical examiner's office and 16 area hospitals. This analysis focused on pediatric sudden death (ages 0-17).

\section{Postmortem examination}

For all cases in which an autopsy was conducted, detailed information, including results of cardiac pathologic examination and toxicology screen were obtained. Detailed criteria for cardiac pathologic diagnosis have been published previously (5).

\section{In-house adjudication of SCA cases}

By evaluating all available medical records, a comprehensive evaluation was performed for each case of presumed SCA, including analysis of circumstances of death, medical records and available autopsy data. All collected clinical information was assembled in a relational database. A process of in-house adjudication was employed to determine cases meeting criteria for SCA. Independent assessments were made by three clinicians, followed by a consensus review. In the event of a disagreement regarding a specific case, the determination was based on the majority opinion. 


\section{Pediatric sudden death cases}

Pediatric sudden deaths were adjudicated in the same manner as adult cases of SCA. In addition, we assigned a SIDS phenotype to infants with a sudden unexplained death before age one. For all such cases, a detailed postmortem examination was performed to confirm that an etiology of sudden death could not be determined. A standard definition of SIDS was employed (6)"the sudden death of an infant under 1 year of age, which remains unexplained after a thorough case investigation, including performance of a complete autopsy, examination of the death scene, and a review of the clinical history."

\section{Statistical Methods}

Descriptive statistics were produced for the population of pediatric sudden death using means and standard deviations for continuous variables and frequencies for categorical variables. Annual incidence rates were calculated by summing the total SCA cases in children aged 0-17 in Multnomah County over three years, dividing by three to produce an average annual incidence, and dividing by the total county population using US Census year 2000 data $(\mathrm{n}=660,486$ for the rate per 100,000 population $)$ and the total pediatric population $(\mathrm{n}=147,250$ for the rate per 100,000 children). Ninety-five percent confidence intervals for the rates were calculated using confidence limit factors for a Poisson-distributed variable (7). We also calculated the number of SIDS cases per 1,000 live births, using live birth counts from the National Center for Health Statistics, Division of Health Statistics (2000).

\section{RESULTS}

\section{Annual incidence of sudden death in children}

During 2002-2005, a total of 33 children met criteria for sudden death. Overall, the median age was $0.37 \mathrm{yr}$, with an age range of $0.03-12.3$ years. A slight majority of the group was female $(58 \%)$, but this difference was not significant $(\mathrm{p}=0.76)$. The gender and age-based composition of prospectively determined sudden death cases is shown in the Table.

The pediatric annual incidence rate per 100,000 population was 1.7 (95\% C.I. $1.1-2.3$ ), compared to 60/100,000 for all ages (Table 1). The pediatric annual incidence rate per 100,000 children was 7.5 (95\% C.I. 5.1 - 10.5). The age-specific annual incidence rate for infants age $<1$ who suffered a sudden death was $96.0 / 100,000$. Pediatric sudden death constituted $2.8 \%$ of all SCAs that occurred during this period.

\section{Detailed description and etiology of pediatric sudden death}

The majority of sudden deaths $(\mathrm{n}=25 ; 76 \%)$ occurred in children age $<1 \mathrm{yr}$. Autopsies had been performed on all infants, and physician records were available for $88 \%$. A total of 23 cases ( $92 \%$ of all infants) met criteria for SIDS. The gender distribution of SIDS cases was similar to that in all pediatric cases, with a non-significant trend toward predominance of females $(57 \%, \mathrm{p}=0.53)$. For these SIDS cases, the annual incidence rate was 0.8 per 1,000 live births. For 22 of the SIDS cases, information was available to assess prior history or symptoms. When available, prior medical history was varied (e.g., history of breath holding $\mathrm{n}=2$; apnea and previous near-SIDS episode $n=1$; asthma $n=1$; methadone addiction in the mother $n=1$ ). Current illness symptoms were noted in $14(64 \%)$ of the subjects, but were predominantly mild (cold $\mathrm{n}=8$; fever/virus $\mathrm{n}=2$; pertussis $\mathrm{n}=2$; bronchiolitis $\mathrm{n}=1$ ).

Among the SIDS cases, smoking in the home was noted for $41 \%$ of subjects (though smoking circumstances were unknown for another $41 \%$ ). Fifty-nine percent of cases were white nonHispanic, 27\% were black, and one case (4.6\%) each were American Indian/Alaskan Native, Asian, and white Hispanic. 
Only two of the 25 infants with sudden death did not meet criteria for SIDS. Both had congenital heart defects identified at autopsy (an interrupted aortic arch; and a ventricular septal defect, aortic stenosis, and non-compaction of the left ventricle); their deaths occurred within the first three weeks of life.

Among the eight children age $\geq 1$, five had an autopsy available and seven had physician records available for review. Four of the eight children (ages $2-8 \mathrm{yrs}$ ) had a history of chronic seizure disorder, but all died in their sleep. Two had congenital heart disease (severe Ebstein's anomaly with Wolff-Parkinson-White Syndrome according to medical history (age 12); and hypertrophic cardiomyopathy with subaortic stenosis and a family history of sudden death (age 11)).

\section{Location of Events}

Half of the infants who met our criteria for SIDS were sleeping in a shared bed or couch at the time of their sudden death (Table 2). Forty-one percent were sleeping in a crib, one infant (5\%) was sleeping in a bouncy chair, and one infant was sleeping in an unspecified location. One of the two infants with a congenital defect was asleep; the other was awake.

Among the 22 SIDS cases with information available, about one-quarter (27\%) were found in the supine position, while $41 \%$ were found in the prone position, and $23 \%$ on their sides (Table 2). One infant (5\% of the total) was sitting in a bouncy chair; the sleeping position of one infant was not recorded.

Among the eight children age $\geq 1$, location of death varied. All four children with a history of seizure disorder (ages 2-8) died while sleeping; one of these children also had cerebral palsy. Both children with known congenital heart disease (ages 11-12) died while participating in athletic activities. Of the remaining two, one (age 1) died after suffering a sudden collapse with VF noted at the time of attempted resuscitation, and the other (age 2) died while sleeping following a recent febrile illness.

\section{Details of attempted resuscitation}

Resuscitation was attempted in $64 \%$ of pediatric cases. Information regarding the presenting rhythm was available for $22(67 \%)$ of the 33 children. The majority of these children were found to be in asystole ( 20 of 22; 91\%). Two children (9\%) presented with ventricular fibrillation. One of these two children had return of spontaneous circulation, as did another child found in asystole, but none of the children survived to be discharged from the hospital. In comparison, for adults the return of spontaneous circulation rate was $24 \%$ and the survival rate was at least $8 \%$.

\section{DISCUSSION}

\section{Summary of Main Findings}

Our study found that pediatric sudden death was a small subgroup (2.8\%) of all SCAs in the population during a three year time frame, with an annual incidence of 1.7/100,000 population. Previous studies have noted an annual incidence of pediatric sudden death varying from 0.8 to $6.2 / 100,000$ (1-3). Our results indicate that the true incidence is closer to the lower estimate and are in agreement with the study by Driscoll and Edwards who reported an annual incidence of 1.3/100,000 from Olmsted County, Minnesota. This retrospective study evaluated pediatric sudden death incidence during 1950-1982 in a significantly smaller population sample. As we have reported earlier, retrospective designation of SCA based on death certificates has the potential for significant error (4). A strength of the current study is that this is likely to be the first prospective population-based study to determine an incidence for pediatric sudden death. 
The preponderance of SIDS among pediatric sudden deaths in this population is of interest and at $0.8 / 1000$ live births, constituted the majority of sudden deaths in children. Autopsy series in the young have previously shown high rates of sudden death among neonates compared to older children (8) and our findings have confirmed this observation in a prospective populationbased study.

Our study also found that SIDS is the most common cause of sudden death among children. SIDS is a poorly understood condition/process and may be due to a multiplicity of factors. Somewhat contrary to the published literature (9), while this was a statistically non-significant finding, we observed female cases to be more common than males (57\% female). It is difficult to ascertain whether this can be attributed to the racial/ethnic composition or cultural/ behavioral traits of the local population or whether this trend would have reversed with a larger number of SIDS cases. Consistent with the published literature for SIDS, the prone or side sleeping positions, bed-sharing and cigarette smoking were identified as the most common associations in the present study $(10,11)$. Similar to previously reported studies, SIDS cases in the present study were more common in African American infants (27\% of SIDS cases were of African American race, while African Americans make up approximately 6\% of the county population) (9). Behavioral measures such as avoidance of prone and side positions for infant sleep, smoke exposure, soft bedding and overheating have been found to be of proven utility in decreasing the incidence of SIDS $(9,12)$. An early case report identified a molecular link between cardiac channelopathies and SIDS (13), and findings of mutations in genes that cause the long QT syndrome among SIDS cases have been confirmed in subsequent larger studies $(14,15)$. Taken together, existing studies would suggest that approximately $10 \%$ of SIDS cases are attributable to the congenital long QT syndrome. We did not perform a "genetic autopsy" $(16,17)$ and are unable to shed any light on this aspect of the SIDS discussion. In infants, the second most common cause of sudden death (after SIDS) was undiagnosed congenital heart disease noted in two babies. Both these patients had lesions that could have been diagnosed by routine physical examination. One patient had aortic stenosis, ventricular septal defect and non-compaction of the left ventricle. The other infant had an interrupted aortic arch.

An early paper from Schwartz and colleagues put forward the concept of neonatal screening using 12-lead ECGs for prevention of long QT syndrome (18). However, this approach has been vigorously debated over the years. ECG screening was performed on the third day of life in 34,442 newborns (19). Of these, 24 became SIDS cases and had a significantly longer mean QTc compared to the remainder. The odds ratio for SIDS in newborns with QTc $>440 \mathrm{~ms}$ was 41.3 (95\% confidence interval 17.3-98.4). Proponents of newborn ECG screening argue that this screening methodology should be applied broadly for detection of long QT syndrome since it is cost effective [the cost per year-of-life saved between $€ 7,400$ and $€ 20,400$ (20)] and has the additional potential to detect other forms of congenital heart disease $(20,21)$. Opponents of neonatal screening make the case that there are costs related to false positive results (e.g. counseling of parents), that pharmacological therapy may not be effective in the newborn, that the false positive rate is significant and that genetic testing is yet not universally available (22). While the present study was not designed with a focus on screening, the preponderance of SIDS would suggest that if screening is conducted in the young, it is likely to be more helpful in neonates rather than older children.

The majority of children were found to be in asystole by the first responders. It is important to recognize that the acute event may initially have manifested with ventricular tachycardia or ventricular fibrillation and degenerated to asystole over time. While this cannot be confirmed, one possibility is that SIDS, affecting the majority of subjects in this study, occurs at times and circumstances that delay the emergency response time. 


\section{Conclusions}

In this population-based study, the overall incidence of pediatric sudden death was low (3\% of total), but SIDS constituted the majority of sudden deaths in children (70\% overall, 0.8/1000 live births). Population education to prevent SIDS and emphasis on the role of enhanced postnatal evaluation to diagnose occult heart disease are the two interventions that may have the greatest impact on prevention of sudden death in children.

\section{Acknowledgments}

The authors would like to acknowledge the significant contribution of American Medical Response, Portland/Gresham fire departments, the Multnomah County Medical Examiner's office and the emergency medicine, cardiology and primary care physicians and allied health personnel of the 16 area hospitals.

Funded, in part, by National Heart Lung and Blood Institute R01HL088416 to Dr Chugh. Dr Chugh is the Pauline and Harold Price Endowed Professor of Cardiac Electrophysiology at the Cedars-Sinai Medical Center, Los Angeles, CA.

\section{References}

1. Driscoll DJ, Edwards WD. Sudden unexpected death in children and adolescents. J Am Coll Cardiol 1985;5:118B-121B. [PubMed: 3964798]

2. Neuspiel DR, Kuller LH. Sudden and unexpected natural death in childhood and adolescence. Jama 1985;254:1321-5. [PubMed: 4021009]

3. Wren C. Sudden death in children and adolescents. Heart 2002;88:426-31. [PubMed: 12231612]

4. Chugh SS, Jui J, Gunson K, et al. Current burden of sudden cardiac death: multiple source surveillance versus retrospective death certificate-based review in a large U.S. community. J Am Coll Cardiol 2004;44:1268-75. [PubMed: 15364331]

5. Chugh SS, Kelly KL, Titus JL. Sudden cardiac death with apparently normal heart. Circulation 2000;102:649-54. [PubMed: 10931805]

6. Willinger M, James LS, Catz C. Defining the sudden infant death syndrome (SIDS): deliberations of an expert panel convened by the National Institute of Child Health and Human Development. Pediatr Pathol 1991;11:677-84. [PubMed: 1745639]

7. dos Santos Silva, I. Cancer Epidemiology: Principles and Methods. Lyon, France: IARC Press; 1999. p. 120

8. Steinberger J, Lucas RV Jr, Edwards JE, et al. Causes of sudden unexpected cardiac death in the first two decades of life. Am J Cardiol 1996;77:992-5. [PubMed: 8644651]

9. Moon RY, Horne RS, Hauck FR. Sudden infant death syndrome. Lancet 2007;370:1578-87. [PubMed: 17980736]

10. Berger S, Dhala A, Friedberg DZ. Sudden cardiac death in infants, children, and adolescents. Pediatr Clin North Am 1999;46:221-34. [PubMed: 10218071]

11. Loghmanee DA, Weese-Mayer DE. Sudden infant death syndrome: another year of new hope but no cure. Curr Opin Pulm Med 2007;13:497-504. [PubMed: 17901755]

12. Dwyer T, Ponsonby AL. Sudden infant death syndrome and prone sleeping position. Ann Epidemiol 2009;19:245-9. [PubMed: 19344862]

13. Schwartz PJ, Priori SG, Dumaine R, et al. A molecular link between the sudden infant death syndrome and the long-QT syndrome. N Engl J Med 2000;343:262-7. [PubMed: 10911008]

14. Ackerman MJ, Siu BL, Sturner WQ, et al. Postmortem molecular analysis of SCN5A defects in sudden infant death syndrome. Jama 2001;286:2264-9. [PubMed: 11710892]

15. Arnestad M, Crotti L, Rognum TO, et al. Prevalence of long-QT syndrome gene variants in sudden infant death syndrome. Circulation 2007;115:361-7. [PubMed: 17210839]

16. Chugh SS, Senashova O, Watts A, et al. Postmortem molecular screening in unexplained sudden death. J Am Coll Cardiol 2004;43:1625-9. [PubMed: 15120823]

17. Tester DJ, Ackerman MJ. The role of molecular autopsy in unexplained sudden cardiac death. Curr Opin Cardiol 2006;21:166-72. [PubMed: 16601452] 
18. Schwartz PJ, Segantini A. Cardiac innervation, neonatal electrocardiography, and SIDS. A key for a novel preventive strategy? Ann N Y Acad Sci 1988;533:210-20. [PubMed: 3421627]

19. Schwartz PJ, Stramba-Badiale M, Segantini A, et al. Prolongation of the QT interval and the sudden infant death syndrome. N Engl J Med 1998;338:1709-14. [PubMed: 9624190]

20. Quaglini S, Rognoni C, Spazzolini C, Priori SG, Mannarino S, Schwartz PJ. Cost-effectiveness of neonatal ECG screening for the long QT syndrome. Eur Heart J 2006;27:1824-32. [PubMed: 16840497]

21. Schwartz PJ. Pro: Newborn ECG screening to prevent sudden cardiac death. Heart Rhythm 2006;3:1353-5. [PubMed: 17074644]

22. van Langen IM, Wilde AA. Con: Newborn screening to prevent sudden cardiac death? Heart Rhythm 2006;3:1356-9. [PubMed: 17074645]

\section{Abbreviations list}

SCA Sudden cardiac arrest

Ore-SUDS The Oregon Sudden Unexpected Death Study

$\mathrm{VF}$

ventricular fibrillation

SIDS sudden infant death syndrome 
Table 1

Pediatric sudden death cases in Multnomah County, Oregon, February 1, 2002 to January 31, 2005

\begin{tabular}{|c|c|}
\hline & Total Cases $(n=33)$ \\
\hline \multicolumn{2}{|l|}{ Gender } \\
\hline Male & $14(42 \%)$ \\
\hline Female & $19(58 \%)$ \\
\hline \multicolumn{2}{|l|}{ Age at time of arrest } \\
\hline$<1$ year & $25(76 \%)$ \\
\hline $1-4$ & $3(9 \%)$ \\
\hline $5-9$ & $3(9 \%)$ \\
\hline $10-14$ & $2(6 \%)$ \\
\hline $15-17$ & 0 \\
\hline Median age (yrs) & 0.37 \\
\hline Age range, yrs (min - max) & $0.03-12.3$ \\
\hline Annual pediatric incidence rate per 100,000 population $(95 \% \mathrm{CI})^{*}$ & $1.7(1.1-2.3)$ \\
\hline Annual pediatric incidence rate per 100,000 children $(95 \% \mathrm{CI}){ }^{*}$ & $7.5(5.1-10.5)$ \\
\hline \multicolumn{2}{|l|}{ Annual incidence rate per 100,000 children $(95 \% \mathrm{CI})^{*}$, by age category } \\
\hline$<1$ & $96.0(62.1-142.0)$ \\
\hline $1-4$ & $3.0(0.6-8.7)$ \\
\hline $5-9$ & $2.4(0.5-7.1)$ \\
\hline $10-14$ & $1.7(0.2-6.0)$ \\
\hline \multicolumn{2}{|l|}{ Cause of death among infants $(n=25)$} \\
\hline $\operatorname{SIDS}^{\dagger}$ & $23(92 \%)$ \\
\hline Congenital heart disease $\mathrm{e}^{t}$ & $2(8 \%)$ \\
\hline Annual incidence of SIDS/1000 live births & 0.8 \\
\hline \multicolumn{2}{|l|}{ Autopsy findings or medical history, ages $1-17(n=8)$} \\
\hline Chronic seizure disorder & $4(50 \%)$ \\
\hline Congenital heart disease ${ }^{\S}$ & $2(25 \%)$ \\
\hline Other & $2(5 \%)$ \\
\hline History of seizure disorder & $4(12 \%)$ \\
\hline
\end{tabular}




\section{Table 2}

Circumstances of sudden death among infants age $<1$ year meeting criteria for sudden infant death syndrome (SIDS) in Multnomah County, Oregon, February 1, 2002 to January 31, 2005

\begin{tabular}{lc}
\hline & Total Cases (n= 23) \\
\hline Gender & $10(43 \%)$ \\
Male & $13(57 \%)$ \\
Female & \\
Place found ${ }^{*}$ & $9(41 \%)$ \\
Crib & $9(41 \%)$ \\
Shared bed & $2(9 \%)$ \\
Couch & $1(5 \%)$ \\
Bouncy chair & $1(5 \%)$ \\
Not specified & \\
Position found & \\
Supine & $6(27 \%)$ \\
Prone & $9(41 \%)$ \\
Side & $5(23 \%)$ \\
Sitting & $1(5 \%)$ \\
Not specified & $1(5 \%)$ \\
\hline Data missing for one SIDS case with unavailable history and circumstances. & \\
&
\end{tabular}

